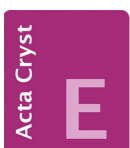

CRYSTALLOGRAPHIC COMMUNICATIONS

ISSN 2056-9890

Received 22 January 2020

Accepted 19 February 2020

Edited by M. Weil, Vienna University of Technology, Austria

Keywords: crystal structure; polymorphism; pyridazine; Hirshfeld surface analysis.

CCDC references: 1985197; 1985196

Supporting information: this article has supporting information at journals.iucr.org/e

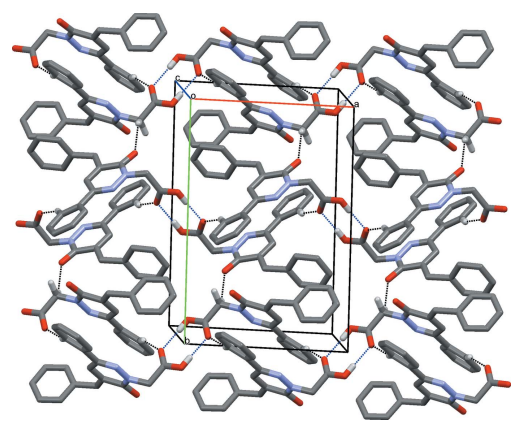

OPEN $\odot$ ACCESS

\section{Polymorphism of 2-(5-benzyl-6-oxo-3-phenyl-1,6- dihydropyridazin-1-yl)acetic acid with two monoclinic modifications: crystal structures and Hirshfeld surface analyses}

\author{
Said Daoui, ${ }^{a} *$ Cemile Baydere, ${ }^{b *}$ Tarik Chelfi, ${ }^{a}$ Fouad El Kalai, ${ }^{a}$ Necmi Dege, \\ Khalid Karrouchic ${ }^{\mathrm{c}}$ and Noureddine Benchat ${ }^{\mathrm{a}}$
}

\footnotetext{
aLaboratory of Applied Chemistry and Environment (LCAE), Faculty of Sciences, Mohamed I University, 60000 Oujda, Morocco, 'bepartment of Physics, Faculty of Arts and Sciences, Ondokuz Mayıs University, 55139-Samsun, Turkey, and ${ }^{\mathrm{c}}$ Laboratory of Medicinal Chemistry, Faculty of Medicine and Pharmacy, University, Mohammed V, Rabat, Morocco. *Correspondence e-mail: saiddaoui62@gmail.com, cemle28baydere@hotmail.com
}

Two polymorphs of the title compound, $\mathrm{C}_{19} \mathrm{H}_{16} \mathrm{~N}_{2} \mathrm{O}_{3}$, were obtained from ethanolic (polymorph I) and methanolic solutions (polymorph II), respectively. Both polymorphs crystallize in the monoclinic system with four formula units per cell and a complete molecule in the asymmetric unit. The main difference between the molecules of (I) and (II) is the reversed position of the hydroxy group of the carboxylic function. All other conformational features are found to be similar in the two molecules. The different orientation of the $\mathrm{OH}$ group results in different hydrogen-bonding schemes in the crystal structures of (I) and (II). Whereas in (I) intermolecular $\mathrm{O}-\mathrm{H} \cdots \mathrm{O}$ hydrogen bonds with the pyridazinone carbonyl $\mathrm{O}$ atom as acceptor generate chains with a $C(7)$ motif extending parallel to the $b$-axis direction, in the crystal of (II) pairs of inversionrelated $\mathrm{O}-\mathrm{H} \cdots \mathrm{O}$ hydrogen bonds with an $R_{2}^{2}(8)$ ring motif between two carboxylic functions are found. The intermolecular interactions in both crystal structures were analysed using Hirshfeld surface analysis and two-dimensional fingerprint plots.

\section{Chemical context}

Pyridazin-3(2H)-ones are an important family of heterocycles because of their great chemical reactivity (Chelfi et al., 2015; Zarrouk et al., 2010), with new products reported recently (Chakraborty et al., 2018; El Kalai et al., 2019a). In addition, the importance of pyridazinones in medicinal chemistry has increased in recent years thanks to their pharmacological properties, including anticancer (Yarden \& Caldes, 2013), antihypertensive (Siddiqui et al., 2011), antibacterial (Akhtar et al., 2016), anti-HIV (Livermore et al., 1993), anti-inflammatory (Singh et al., 2017), antidepressant (Boukharsa et al., 2016), anti-convulsant (Partap et al., 2018) and cardiotonic (Costas et al., 2015) activities. Several pyridazinone-based products are already present in the pharmaceutical market such as Minaprine (Sotelo et al., 2003), Azanrinone (Mahmoodi et al., 2014), Indolidan (Abouzid et al., 2008) and Levosimendan (Archan \& Toller, 2008).

In a continuation of our recent work on the synthesis and crystal structures of new pyridazin-3(2H)-one derivatives (El Kalai et al., 2019b; Daoui et al., 2019a,b), we report here the synthesis, crystal structure and polymorphism of 2-(5-benzyl6-oxo-3-phenylpyridazin-1 $(6 H)$-yl)acetic acid, which is going to be subjected to further pharmacological investigations. 
<smiles>O=C(O)Cn1nc(-c2ccccc2)cc(Cc2ccccc2)c1=O</smiles>

\section{Structural commentary}

The title compound is dimorphic with two monoclinic polymorphs. The molecular structure of polymorph (I) is shown in Fig. 1 and that of polymorph (II) in Fig. 2. The differences in the conformations of the two molecules is shown in the structural overlap drawing (Fig. 3). The main difference between (I) and (II) pertains to the $\mathrm{OH}$ function of the carboxyl group, which is reversed in the two molecules. All other conformational features are quite similar in the molecules of the two polymorphs. In (I), the phenyl ring (C1-C6) and the pyridazine ring $(\mathrm{N} 1 / \mathrm{N} 2 / \mathrm{C} 10-\mathrm{C} 7)$ are nearly co-planar, making a dihedral angle of $5.92(2)^{\circ}$ whereas the phenyl ring of the benzyl group (C14-C19) is perpendicular to the pyridazine ring, with a dihedral angle of $89.91(1)^{\circ}$ (Fig. 1). In (II),

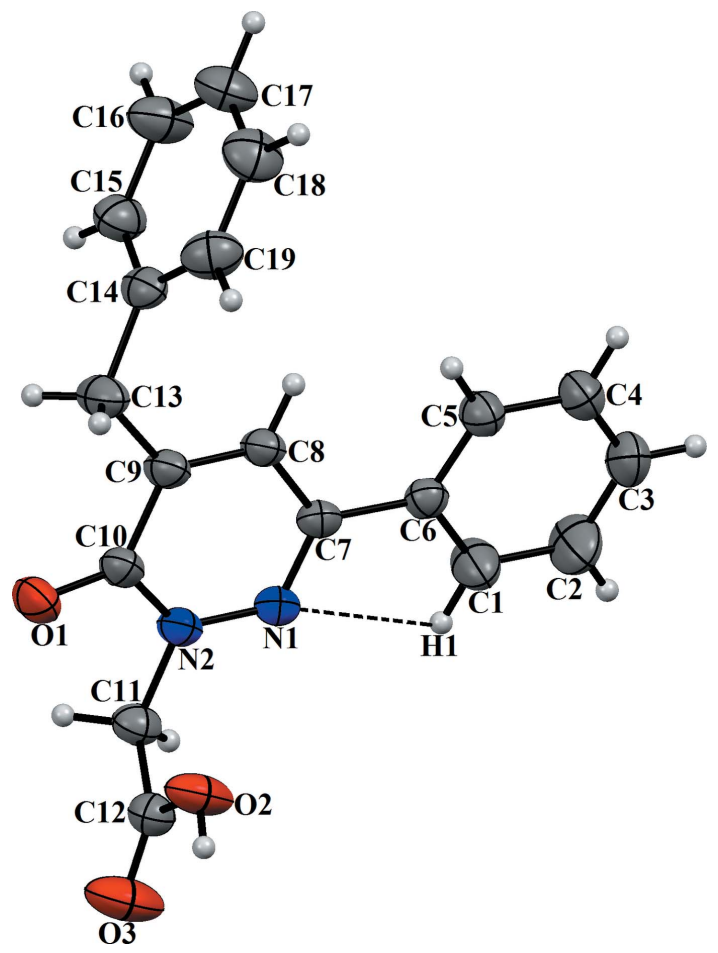

Figure 1

The molecular structure of (I) with displacement ellipsoids drawn at the $30 \%$ probability level.

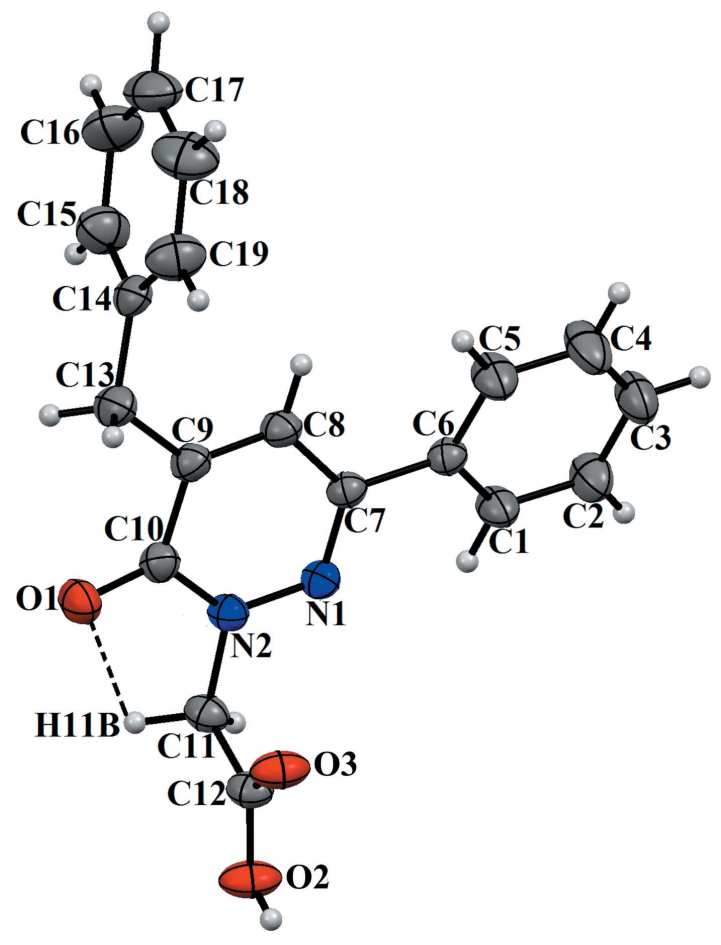

Figure 2

The molecular structure of (II) with displacement ellipsoids drawn at the $30 \%$ probability level.

the corresponding values are $15.44(2)$ and $89.13(1)^{\circ}$, respectively. In the molecule of (I), the carboxyl group has a C12-O 2 bond length of 1.277 (2) $\AA$ between the $\mathrm{C}$ atom and the $\mathrm{OH}$ function, and the $\mathrm{C} 12=\mathrm{O} 3$ bond length of the carbonyl group is 1.187 (2) $\AA$. The corresponding values in (II) are 1.3057 (16) and 1.2108 (18) $\AA$. The differences in the

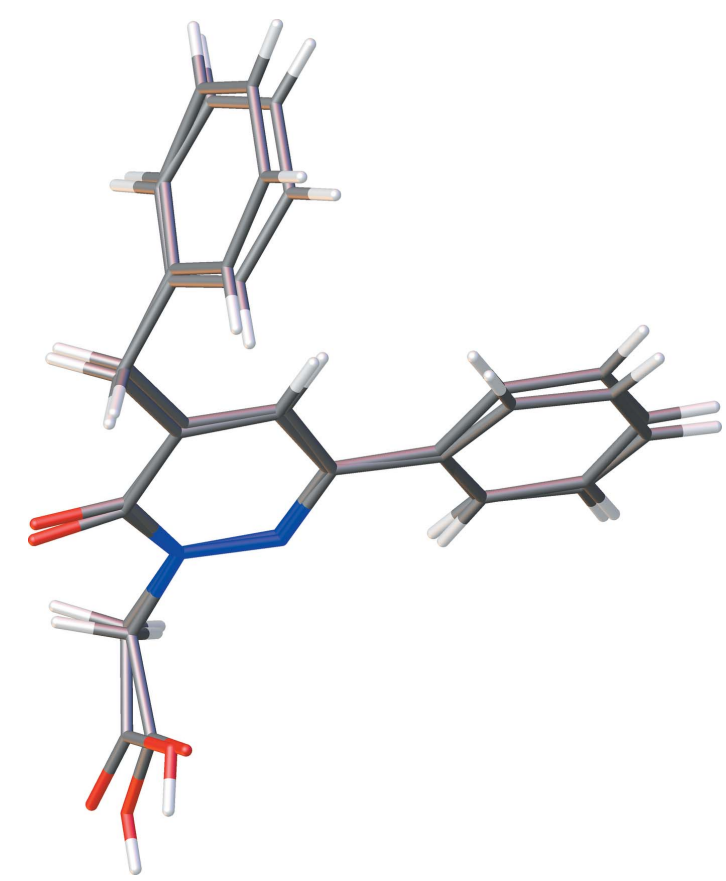

Figure 3

Structural overlap of molecules (I) and (II). 
Table 1

Hydrogen-bond geometry $\left(\AA{ }^{\circ}\right)$ for $\mathbf{I}$.

\begin{tabular}{lllll}
\hline$D-\mathrm{H} \cdots A$ & $D-\mathrm{H}$ & $\mathrm{H} \cdots A$ & $D \cdots A$ & $D-\mathrm{H} \cdots A$ \\
\hline $\mathrm{O} 2-\mathrm{H} 2 \cdots \mathrm{O} 1^{\mathrm{i}}$ & 0.82 & 1.82 & $2.593(2)$ & 156 \\
$\mathrm{C} 1-\mathrm{H} 1 \cdots \mathrm{N} 1$ & 0.93 & 2.47 & $2.780(3)$ & 100 \\
\hline
\end{tabular}

Symmetry code: (i) $-x+\frac{3}{2}, y-\frac{1}{2},-z+\frac{1}{2}$.

bond lengths of the two carboxylic groups can be attributed to their different roles in intermolecular hydrogen bonding (see below). In both molecules, weak intramolecular hydrogen bonds $[\mathrm{C}-\mathrm{H} \cdots \mathrm{N}$ for (I) and $\mathrm{C}-\mathrm{H} \cdots \mathrm{O}$ for (II); Figs. 1 and 2, Tables 1 and 2] stabilize the molecular conformation.

\section{Supramolecular features}

In the crystal structure of (I), molecules are linked by $\mathrm{O} 2-$ $\mathrm{H} 2 \cdots \mathrm{O} 1^{\mathrm{i}}$ hydrogen bonds between the carboxylic $\mathrm{OH}$ function and the pyridazinone carbonyl $\mathrm{O} 1$ atom of a neighbouring molecule, generating $C(7)$ chains extending parallel to the $b$ axis direction (Fig. 4, Table 1 ). A weak $\pi-\pi$ stacking interaction occurs between the pyridazinone rings of inversionrelated molecules $[C g 1 \cdots C g 1(1-\mathrm{x}, 1-\mathrm{y}, 1-\mathrm{z})]$, with a centroid-to-centroid distance of 3.8437 (12) $\AA$ and a slippage of 1.690 ( $C g 1$ is the centroid of the N1/N2/C10-C7 ring) (Fig. 4). As a result of the reversed orientation of the carboxylic hydroxy function, in the crystal structure of (II) the hydrogen-bonding scheme is different. Here molecules are linked by pairs of $\mathrm{O} 3-\mathrm{H} 3 \cdot \mathrm{O} 2{ }^{\mathrm{i}}$ hydrogen bonds between the carboxylic groups of neighbouring molecules, forming inversion dimers with an $R_{2}^{2}(8)$ ring motif. The dimers are linked by weak $\mathrm{C} 5-\mathrm{H} 5 \cdots \mathrm{O} 2^{\mathrm{ii}}$ and $\mathrm{C} 11-\mathrm{H} 11 A \cdots \mathrm{O} 1^{\mathrm{iii}}$ hydrogen bonds,

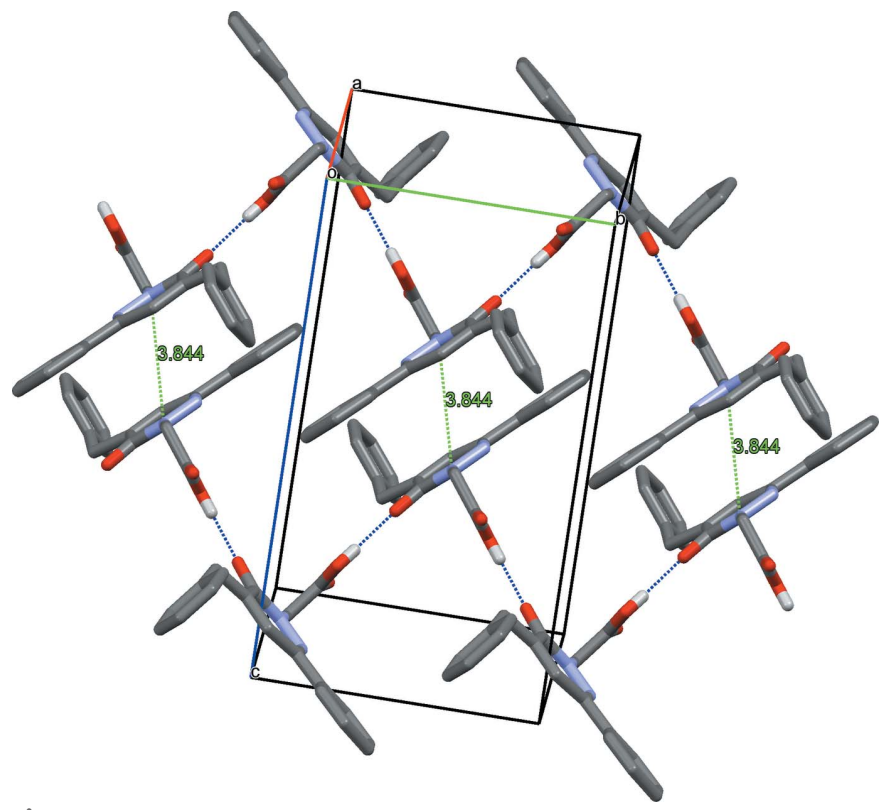

Figure 4

The crystal packing of (I). The $\mathrm{O}-\mathrm{H} \cdots \mathrm{O}$ hydrogen bonds are shown as blue dotteded lines, and $\pi-\pi$ contacts are represented by green dotted lines. For clarity, only $\mathrm{H}$ atoms involved in hydrogen bonding (white sticks) were included.
Table 2

Hydrogen-bond geometry $\left(\AA,^{\circ}\right)$ for II.

\begin{tabular}{lllll}
\hline$D-\mathrm{H} \cdots A$ & $D-\mathrm{H}$ & $\mathrm{H} \cdots A$ & $D \cdots A$ & $D-\mathrm{H} \cdots A$ \\
\hline $\mathrm{C} 11-\mathrm{H} 11 B \cdots \mathrm{O} 1$ & 0.97 & 2.39 & $2.7325(19)$ & 100 \\
$\mathrm{O}^{2}-\mathrm{H} 3 \cdots \mathrm{O} 3^{\mathrm{i}}$ & 0.82 & 1.84 & $2.6599(16)$ & 177 \\
$\mathrm{C} 5-\mathrm{H} 5 \cdots 3^{\mathrm{ii}}$ & 0.93 & 2.40 & $3.280(2)$ & 159 \\
$\mathrm{C}^{1}-\mathrm{H} 11 A \cdots \mathrm{O} 1{ }^{\mathrm{iii}}$ & 0.97 & 2.47 & $3.2814(19)$ & 141 \\
\hline
\end{tabular}

Symmetry codes: (i) $-x+2,-y+1,-z+1$; $\quad$ (ii) $-x+1,-y+1,-z+1$; (iii) $x,-y+\frac{1}{2}, z-\frac{1}{2}$.

forming $C(8)$ chains extending parallel to the $b$-axis direction (Table 2, Fig. 5). The crystal packing of (II) also features weak $\pi-\pi$ interactions involving the centroids of the N1/N2/C7-C10 $(C g 1)$ and $\mathrm{C} 14-\mathrm{C} 19$ (Cg3) rings, with $C g 1 \cdots C g 3\left(x, \frac{1}{2}-y\right.$, $\left.-\frac{1}{2}+z\right)=4.3830(12) \AA$.

\section{Database survey}

A search of the Cambridge Structural Database (CSD, version 5.40, update August 2019; Groom et al., 2016) using 2-[6oxopyridazin-1(6H)-yl]acetic acid as the main skeleton revealed the presence of three structures similar to the title compound, but with different substituents on the pyridazione ring, viz. ethyl 2-[6-oxo-3,4-diphenyl-1,6-dihydropyridazin-1yl]acetic acid acetate (CIPTOL; Aydin et al., 2007), ethyl 3-methyl-6-oxo-5-[3-(trifluoromethyl)phenyl]-1,6-dihydro-1pyridazineacetate (QANVOR; Xu et al., 2005) and ethyl \{4[(5-chloro-1-benzofuran-2-yl)methyl]-3-methyl-6-oxopyridazin-1(6H)-yl \}acetate (XULSEE; Boukharsa et al., 2015). Like in (I) and (II), the packing within the crystal structures of these compounds is dominated by $\mathrm{O}-\mathrm{H} \cdots \mathrm{O}$ hydrogen bonds and consolidated by $\mathrm{C}-\mathrm{H} \cdots \mathrm{O}$ interactions. In CIPTOL, the pyridazinone ring and two phenyl rings are inclined to each other by 72.73 (11) and $49.97(10)^{\circ}$ compared to the corresponding dihedral angles of $5.92(2), 89.91(1)$ and $15.44(2)^{\circ}$, $89.13(1)^{\circ}$ in (I) and (II), respectively. In QANVOR, the

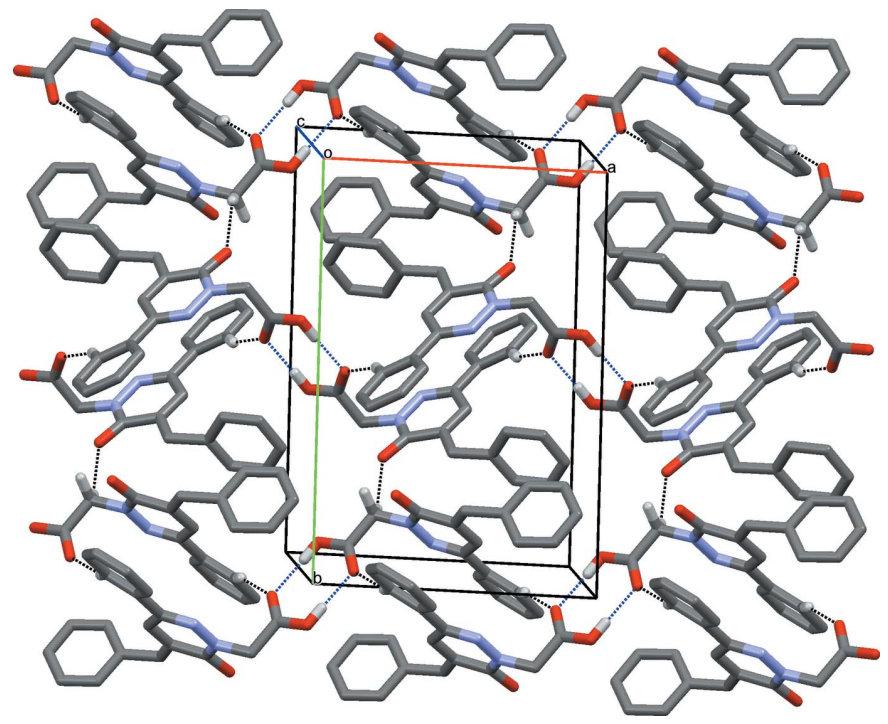

Figure 5

The crystal packing of (II), with $\mathrm{O}-\mathrm{H} \cdots \mathrm{O}$ and $\mathrm{C}-\mathrm{H} \cdots \mathrm{O}$ interactions shown as blue and black dotted lines, respectively. 


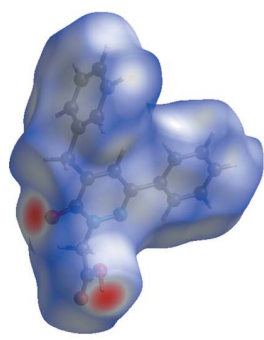

(a)

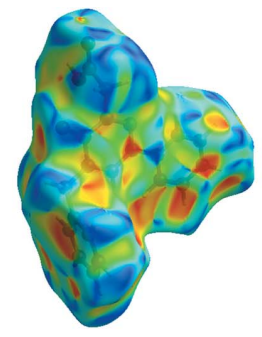

(b)

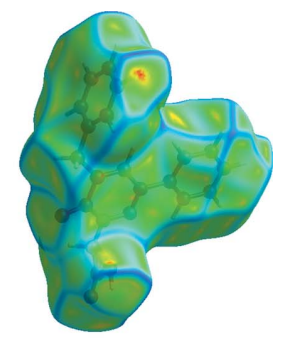

(c)
Figure 6

(a) The Hirshfeld surface of (I) mapped over $d_{\text {norm }}$, and plotted in the range -0.7266 (red) to 1.4843 (blue) a.u.; (b) the Hirshfeld surface mapped over shape-index; $(c)$ the Hirshfeld surface mapped over curvedness.

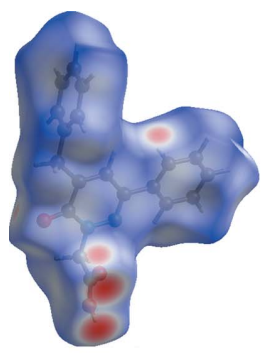

(a)

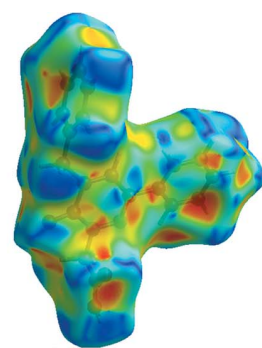

(b)

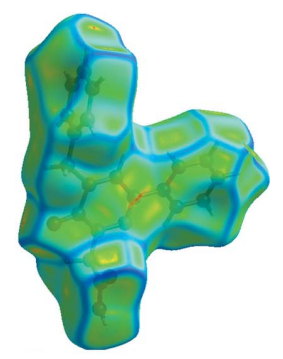

(c)
Figure 7

(a) The Hirshfeld surface of (II) mapped over $d_{\text {norm }}$, and plotted in the range -0.7232 (red) to 1.3047 (blue) a.u.; (b) the Hirshfeld surface mapped over shape-index, $(c)$ the Hirshfeld surface mapped over curvedness.

3-(trifluoromethyl)phenyl and pyridazinone rings are approximately coplanar with a dihedral angle of $4.84(13)^{\circ}$. In XULSEE, the dihedral angle between the benzofuran ring system [maximum deviation 0.014 (2) $\AA$ ] and the pyridazinone ring is $73.33(8)^{\circ}$.

\section{Hirshfeld surface analysis}

Hirshfeld surface analysis was applied to quantify the intermolecular contacts in (I) and (II), using CrystalExplorer17.5 (Turner et al., 2017). A standard (high) surface resolution with the three-dimensional $d_{\text {norm }}$ surfaces plotted over a fixed colour scale of -0.7266 (red) to 1.4843 (blue) a.u. was used for (I) and of -0.7232 (red) to 1.3047 (blue) a.u. for (II). The bright-red spots on the Hirshfeld surface mapped over $d_{\text {norm }}$ show the presence of $\mathrm{O}-\mathrm{H} \cdots \mathrm{O}$ interactions with neighbouring molecules in (I) (Fig. 6a) and (II) (Fig. 7a), respectively. The presence of red and blue triangles on the shapeindex map [Fig. $6 b$ (I) and $7 b$ (II)] are indicative for the presence of $\pi-\pi$ stacking interactions. The curvedness plots show flat surface patches characteristic of planar stacking (Fig. $6 c$ and 7c). The complete two-dimensional fingerprint plots are shown in Fig. $8 a$ and $9 a$ for (I) and (II). The H $\cdots H$, $\mathrm{H} \cdots \mathrm{O}, \mathrm{C} \cdots \mathrm{H}, \mathrm{C} \cdots \mathrm{C}, \mathrm{C} \cdots \mathrm{N}, \mathrm{N} \cdots \mathrm{H}$ and $\mathrm{C} \cdots \mathrm{O}$ interactions are illustrated in Fig. $8 b-h$ for $(\mathbf{I})$, and $\mathrm{H} \cdots \mathrm{H}, \mathrm{C} \cdots \mathrm{H}, \mathrm{H} \cdots \mathrm{O}$, $\mathrm{N} \cdots \mathrm{H}, \mathrm{C} \cdots \mathrm{C}$ and $\mathrm{C} \cdot \mathrm{O}$ interactions are illustrated in Fig. $9 b-$ $g$ for (II). In both crystal structures, $\mathrm{H} \cdots \mathrm{H}$ interactions make the largest contributions to the overall Hirshfeld surfaces
[48.7\% for (I) and $43.6 \%$ for (II)]. As expected from the intermolecular $\mathrm{O}-\mathrm{H} \cdots \mathrm{O}$ and $\mathrm{C}-\mathrm{H} \cdots \mathrm{O}$ contacts detailed in Tables 1 and 2, H. .O contacts also account for a high percentage contributions [21.5\% (I) and $21.9 \%$ (II)] and are indicated by a pair of wings at $d_{\mathrm{e}}+d_{\mathrm{i}} \sim 1.7 \AA$ [Fig. $8 c$ (I) and $9 d$ (II)]. The C $\cdots \mathrm{H}$ contacts, with percentage contributions of $19.2 \%$ in (I) and $22.5 \%$ in (II) appear in the fingerprint plots as two distinct spikes at $d_{\mathrm{e}}+d_{\mathrm{i}} \sim 2.9 \AA$ in (I) and $3.0 \AA$ in (II) (Fig. $8 d$ and 9c). The $\mathrm{C} \cdots \mathrm{C}$ contacts, which refer to $\pi-\pi$

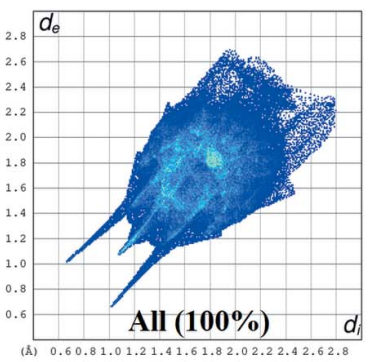

(a)

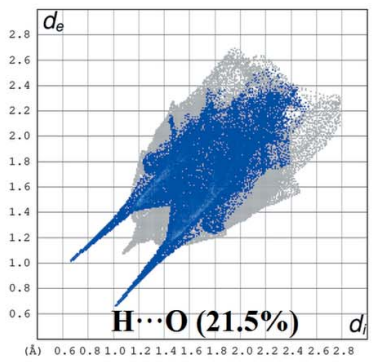

(c)

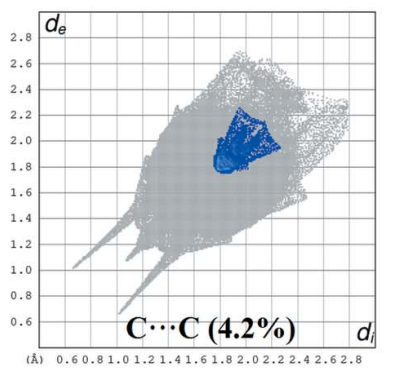

(e)

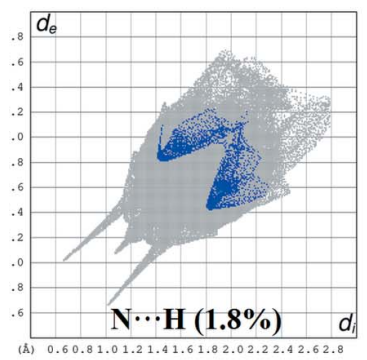

(g)

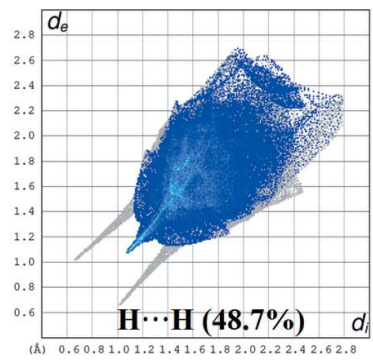

(b)

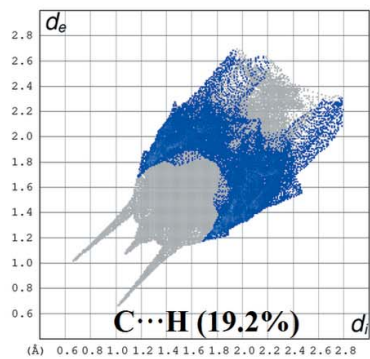

$(d)$

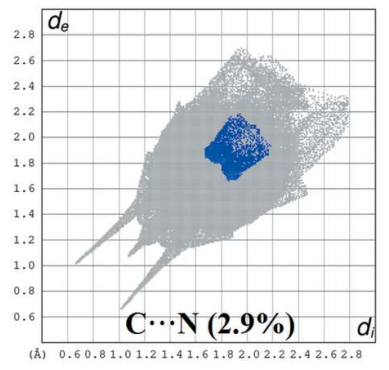

$(f)$

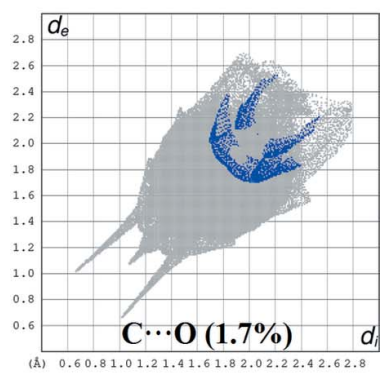

(h)
Figure 8

Two-dimensional fingerprint plots for (I): $(a)$ all intermolecular interactions; (b) $\mathrm{H} \cdots \mathrm{H}$ contacts; $(c) \mathrm{H} \cdots \mathrm{O}$ contacts; $(d) \mathrm{C} \cdots \mathrm{H}$ contacts; $(e)$ C $\cdots \mathrm{C}$ contacts; $(f) \mathrm{C} \cdots \mathrm{N}$ contacts; $(g) \mathrm{N} \cdots \mathrm{H}$ contacts; $(h) \mathrm{C} \cdots \mathrm{O}$ contacts. 


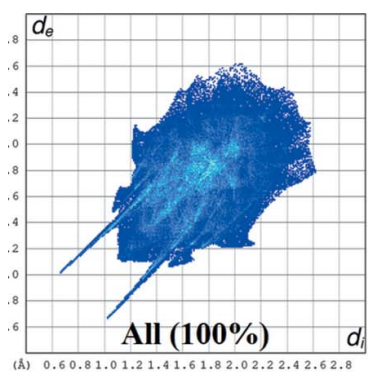

(a)

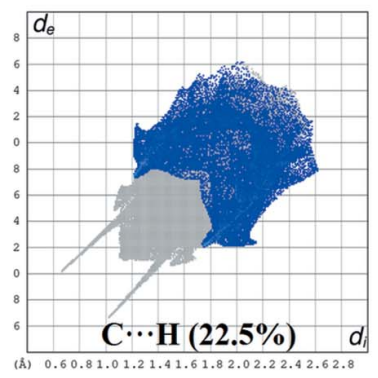

(c)

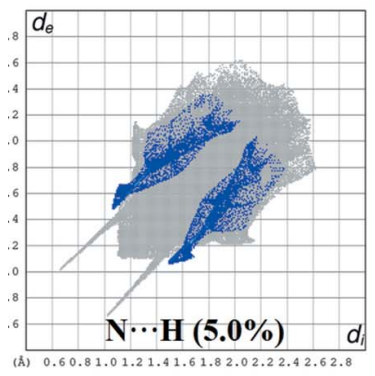

(e)

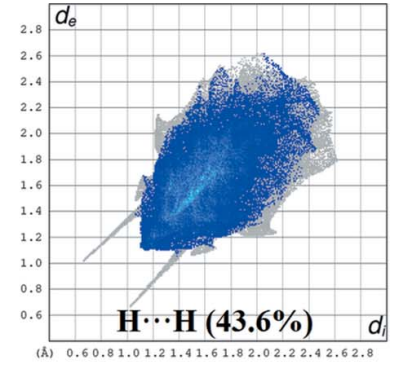

(b)

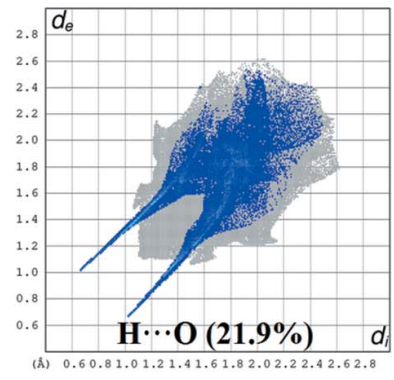

(d)

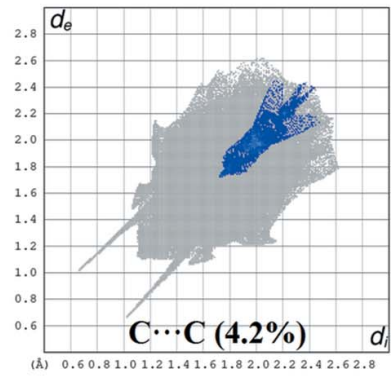

$(f)$

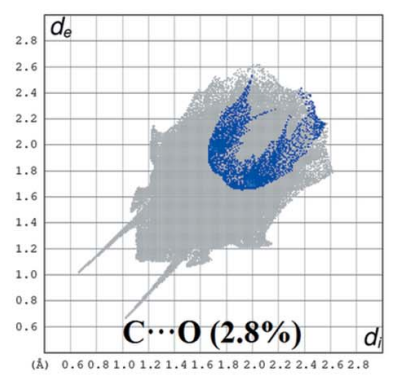

(g)

Figure 9

Two-dimensional fingerprint plots for (II): $(a)$ all intermolecular interactions; (b) $\mathrm{H} \cdots \mathrm{H}$ contacts; (c) $\mathrm{C} \cdots \mathrm{H}$ contacts; $(d) \mathrm{H} \cdots \mathrm{O}$ contacts; $(e)$ $\mathrm{N} \cdots \mathrm{H}$ contacts; $(f) \mathrm{C} \cdots \mathrm{C}$ contacts; $(g) \mathrm{C} \cdots \mathrm{O}$ contacts.

interactions, contribute $4.2 \%$ of the Hirshfeld surfaces for both (I) and (II) (Fig. $8 e$ and 9f). There are additional $\mathrm{N} \cdots \mathrm{H}$ $(5.0 \%)$ and $\mathrm{C} \cdots \mathrm{O}(2.8 \%)$ contacts in (II), while in (I) (where $\mathrm{N} \cdots \mathrm{H}=1.8 \%$ and $\mathrm{C} \cdots \mathrm{O}=1.7 \%), \mathrm{C} \cdot \mathrm{N}(2.9 \%)$ interactions are also observed.

\section{Synthesis and crystallization}

A suspension of ethyl 2-(5-benzyl-6-oxo-3-phenylpyridazin$1(6 H)$-yl)acetate $(3.6 \mathrm{mmol})$, and $6 \mathrm{~N} \mathrm{NaOH}(14.4 \mathrm{mmol})$ in ethanol $(50 \mathrm{ml})$ was stirred at $353 \mathrm{~K}$ for $4 \mathrm{~h}$. The mixture was then concentrated in vacuo, diluted with cold water, and acidified with $6 \mathrm{~N} \mathrm{HCl}$. The final product was filtered off by suction filtration and recrystallized from ethanol or methanol. Single crystals of (I) were obtained by slow evaporation of an ethanolic solution at room temperature, and single crystals of (II) were obtained by slow evaporation of a methanolic solution at room temperature.

\section{Refinement}

Crystal data, data collection and structure refinement details are summarized in Table 3. The atom labelling for molecules of (I) and (II) is identical. In the refinement of (I), SIMU, DELU and ISOR commands were used for atoms C12 and O3. For both structures, hydrogen atoms of the carboxylic group were located in a difference-Fourier map and were refined with a fixed $\mathrm{O}-\mathrm{H}$ distance of $0.82 \AA$ and with $U_{\text {iso }}(\mathrm{H})=$ $1.5 U_{\text {eq }}(\mathrm{O})$. All other hydrogen atoms were placed in calculated positions, with $\mathrm{C}-\mathrm{H}=0.93-0.96 \AA$ and allowed to ride on their parent atoms with $U_{\text {iso }}(\mathrm{H})=1.5 U_{\text {eq }}(\mathrm{C}$-methyl $)$ and $1.2 U_{\text {eq }}(\mathrm{C})$ for other $\mathrm{H}$ atoms.

\section{Acknowledgements}

The authors acknowledge the Faculty of Arts and Sciences, Ondokuz Mayis University, Turkey, for the use of the Stoe IPDS 2 diffractometer (purchased under grant F.279 of the University Research Fund).

\section{References}

Abouzid, K., Abdel Hakeem, M., Khalil, O. \& Maklad, Y. (2008). Bioorg. Med. Chem. 16, 382-389.

Akhtar, W., Shaquiquzzaman, M., Akhter, M., Verma, G., Khan, M. F. \& Alam, M. M. (2016). Eur. J. Med. Chem. 123, 256-281.

Archan, S. \& Toller, W. (2008). Curr. Opin. Anaesthesiol. 21, 78-84.

Aydın, A., Doğruer, D. S., Akkurt, M. \& Büyükgüngör, O. (2007). Acta Cryst. E63, o4522.

Boukharsa, Y., El Ammari, L., Taoufik, J., Saadi, M. \& Ansar, M. (2015). Acta Cryst. E71, o291-o292.

Boukharsa, Y., Meddah, B., Tiendrebeogo, R. Y., Ibrahimi, A., Taoufik, J., Cherrah, Y., Benomar, A., Faouzi, M. E. A. \& Ansar, M. (2016). Med. Chem. Res. 25, 494-500.

Chakraborty, M., Sengupta, D., Saha, T. \& Goswami, S. (2018). J. Org. Chem. 83, 7771-7778.

Chelfi, T., Elaatioui, A., Koudad, M., Benchat, N. \& Hacht, B. (2015). J. Mater. Environ. Sci, 6, 2174-2178.

Costas, T., Costas-Lago, M. C., Vila, N., Besada, P., Cano, E. \& Terán, C. (2015). Eur. J. Med. Chem. 94, 113-122.

Daoui, S., Baydere, C., El Kalai, F., Mahi, L., Dege, N., Karrouchi, K. \& Benchat, N. (2019a). Acta Cryst. E75, 1925-1929.

Daoui, S., Çınar, E. B., El Kalai, F., Saddik, R., Dege, N., Karrouchi, K. \& Benchat, N. (2019b). Acta Cryst. E75, 1880-1883.

El Kalai, F., Baydere, C., Daoui, S., Saddik, R., Dege, N., Karrouchi, K. \& Benchat, N. (2019b). Acta Cryst. E75, 892-895.

El Kalai, F., Chelfi, T., Benchat, N., Hacht, B., Bouklah, M., Elaatiaoui, A., Daoui, S., Allali, M., Ben Hadda, T. \& Almalki, F. (2019a). J. Mol. Struct. 1191, 24-31.

Farrugia, L. J. (2012). J. Appl. Cryst. 45, 849-854.

Groom, C. R., Bruno, I. J., Lightfoot, M. P. \& Ward, S. C. (2016). Acta Cryst. B72, 171-179. 
Table 3

Experimental details.

I

Crystal data

Chemical formula

$M_{\mathrm{r}}$

Crystal system, space group

Temperature $(\mathrm{K})$

$a, b, c(\AA)$

$\beta\left({ }^{\circ}\right)$

$V\left(\AA^{3}\right)$

Z

Radiation type

$\mu\left(\mathrm{mm}^{-1}\right)$

Crystal size (mm)

Data collection

Diffractometer

Absorption correction

$T_{\min }, T_{\max }$

No. of measured, independent and observed $[I>$

$2 \sigma(I)]$ reflections

$R_{\text {int }}$

$(\sin \theta / \lambda)_{\max }\left(\AA^{-1}\right)$

Refinement

$R\left[F^{2}>2 \sigma\left(F^{2}\right)\right], w R\left(F^{2}\right), S$

No. of reflections

No. of parameters

No. of restraints

$\mathrm{H}$-atom treatment

$\Delta \rho_{\max }, \Delta \rho_{\min }\left(\mathrm{e} \AA^{-3}\right)$
II

$\mathrm{C}_{19} \mathrm{H}_{16} \mathrm{~N}_{2} \mathrm{O}_{3}$
320.34
Monoclinic, $P 2_{1} / n$
296
$10.5500(8), 9.3679(6), 16.5606(15)$
$93.886(7)$
$1632.9(2)$
4
$\mathrm{Mo} \mathrm{K \alpha}$
0.09
$0.58 \times 0.43 \times 0.34$

$\mathrm{C}_{19} \mathrm{H}_{16} \mathrm{~N}_{2} \mathrm{O}_{3}$

320.34

Monoclinic, $P 2_{1} / c$

296

10.5976 (6), 15.5500 (7), 10.3731 (7)

$109.120(5)$

$1615.11(17)$

4

Mo $K \alpha$

0.09

$0.77 \times 0.70 \times 0.59$

Stoe IPDS 2

Integration ( $X$-RED32; Stoe \& Cie, 2002)

$0.961,0.981$

12987, 4603, 1989

STOE IPDS 2

Integration ( $X$-RED32; Stoe \& Cie, 2002)

$0.950,0.966$

$12114,4562,2560$

0.039

0.037

0.699

$0.053,0.158,0.89$

4603

$0.049,0.131,0.98$

4562

218

0

19

$\mathrm{H}$-atom parameters constrained

$0.35,-0.34$
$\mathrm{H}$-atom parameters constrained $0.21,-0.21$

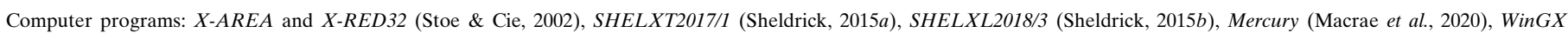
(Farrugia, 2012), PLATON (Spek, 2020) and publCIF (Westrip, 2010).

Livermore, D. G. H., Bethell, R. C., Cammack, N., Hancock, A. P., Hann, M. M., Green, D. V. S., Lamont, R. B., Noble, S. A., Orr, D. C. \& Payne, J. J. (1993). J. Med. Chem. 36, 3784-3794.

Macrae, C. F., Sovago, I., Cottrell, S. J., Galek, P. T. A., McCabe, P., Pidcock, E., Platings, M., Shields, G. P., Stevens, J. S., Towler, M. \& Wood, P. A. (2020). J. Appl. Cryst. 53, 226-235.

Mahmoodi, N. O., Safari, N. \& Sharifzadeh, B. (2014). Synth. Commun. 44, 245-250.

Partap, S., Akhtar, M. J., Yar, M. S., Hassan, M. Z. \& Siddiqui, A. A. (2018). Bioorg. Chem. 77, 74-83.

Sheldrick, G. M. (2015a). Acta Cryst. A71, 3-8.

Sheldrick, G. M. (2015b). Acta Cryst. C71, 3-8.

Siddiqui, A. A., Mishra, R., Shaharyar, M., Husain, A., Rashid, M. \& Pal, P. (2011). Bioorg. Med. Chem. Lett. 21, 1023-1026.

Singh, J., Sharma, D. \& Bansal, R. (2017). J. Heterocycl. Chem. 54, 2935-2945.
Sotelo, E., Coelho, A. \& Raviña, E. (2003). Tetrahedron Lett. 44, 4459-4462.

Spek, A. L. (2020). Acta Cryst. E76, 1-11.

Stoe \& Cie (2002). $X$-AREA and $X$-RED32. Stoe \& Cie GmbH, Darmstadt, Germany.

Turner, M. J., McKinnon, J. J., Wolff, S. K., Grimwood, D. J., Spackman, P. R., Jayatilaka, D. \& Spackman, M. A. (2017). CrystalExplorer17. University of Western Australia. http://hirshfeldsurface.net.

Westrip, S. P. (2010). J. Appl. Cryst. 43, 920-925.

Xu, H., Song, H.-B., Yao, C.-S., Zhu, Y.-Q., Hu, F.-Z., Zou, X.-M. \& Yang, H.-Z. (2005). Acta Cryst. E61, o1561-01563.

Yarden, Y. \& Caldes, C. (2013). Eur. J. Cancer, 49, 2619-2620.

Zarrouk, A., Chelfi, T., Dafali, A., Hammouti, B., Al-Deyab, S. S., Warad, I., Benchat, N. \& Zertoubi, M. (2010). Int. J. Electrochem. Sci. 5, 696-705. 


\section{supporting information}

Acta Cryst. (2020). E76, 432-437 [https://doi.org/10.1107/S2056989020002406]

Polymorphism of 2-(5-benzyl-6-oxo-3-phenyl-1,6-dihydropyridazin-1-yl)acetic acid with two monoclinic modifications: crystal structures and Hirshfeld surface analyses

Said Daoui, Cemile Baydere, Tarik Chelfi, Fouad El Kalai, Necmi Dege, Khalid Karrouchi and Noureddine Benchat

Computing details

For both structures, data collection: $X$-AREA (Stoe \& Cie, 2002); cell refinement: $X$-AREA (Stoe \& Cie, 2002); data reduction: X-RED32 (Stoe \& Cie, 2002); program(s) used to solve structure: SHELXT2017/1 (Sheldrick, 2015a); program(s) used to refine structure: SHELXL2018/3 (Sheldrick, 2015b); molecular graphics: Mercury (Macrae et al., 2020) and PLATON (Spek, 2020); software used to prepare material for publication: WinGX (Farrugia, 2012), PLATON (Spek, 2020) and publCIF (Westrip, 2010).

2-(5-Benzyl-6-oxo-3-phenyl-1,6-dihydropyridazin-1-yl)acetic acid (I)

Crystal data

$\mathrm{C}_{19} \mathrm{H}_{16} \mathrm{~N}_{2} \mathrm{O}_{3}$

$M_{r}=320.34$

Monoclinic, $P 2_{1} / n$

$a=10.5500(8) \AA$

$b=9.3679(6) \AA$

$c=16.5606(15) \AA$

$\beta=93.886(7)^{\circ}$

$V=1632.9(2) \AA^{3}$

$Z=4$

Data collection

Stoe IPDS 2 diffractometer

Radiation source: sealed X-ray tube, 12 x 0.4 $\mathrm{mm}$ long-fine focus

Plane graphite monochromator

Detector resolution: 6.67 pixels $\mathrm{mm}^{-1}$

rotation method scans

Absorption correction: integration

(X-RED32; Stoe \& Cie, 2002)

\section{Refinement}

Refinement on $F^{2}$

Least-squares matrix: full

$R\left[F^{2}>2 \sigma\left(F^{2}\right)\right]=0.053$

$w R\left(F^{2}\right)=0.158$
$F(000)=672$

$D_{\mathrm{x}}=1.303 \mathrm{Mg} \mathrm{m}^{-3}$

Mo $K \alpha$ radiation, $\lambda=0.71073 \AA$

Cell parameters from 9543 reflections

$\theta=1.9-29.8^{\circ}$

$\mu=0.09 \mathrm{~mm}^{-1}$

$T=296 \mathrm{~K}$

Prism, colorless

$0.58 \times 0.43 \times 0.34 \mathrm{~mm}$

$T_{\min }=0.961, T_{\max }=0.981$

12987 measured reflections

4603 independent reflections

1989 reflections with $I>2 \sigma(I)$

$R_{\text {int }}=0.039$

$\theta_{\text {max }}=29.7^{\circ}, \theta_{\min }=2.4^{\circ}$

$h=-12 \rightarrow 14$

$k=-13 \rightarrow 12$

$l=-23 \rightarrow 23$

$S=0.89$

4603 reflections

217 parameters

19 restraints 
Hydrogen site location: inferred from neighbouring sites

$\mathrm{H}$-atom parameters constrained

$$
\begin{gathered}
w=1 /\left[\sigma^{2}\left(F_{\mathrm{o}}^{2}\right)+(0.0772 P)^{2}\right] \\
\text { where } P=\left(F_{\mathrm{o}}^{2}+2 F_{\mathrm{c}}{ }^{2}\right) / 3 \\
(\Delta / \sigma)_{\max }<0.001 \\
\Delta \rho_{\max }=0.35 \mathrm{e} \AA^{-3} \\
\Delta \rho_{\min }=-0.34 \mathrm{e} \AA^{-3}
\end{gathered}
$$

Special details

Geometry. All esds (except the esd in the dihedral angle between two 1.s. planes) are estimated using the full covariance

\begin{tabular}{|c|c|c|c|c|}
\hline & $x$ & $y$ & $z$ & $U_{\text {iso }} * / U_{\text {eq }}$ \\
\hline N2 & $0.63345(14)$ & $0.43785(17)$ & $0.40115(9)$ & $0.0550(4)$ \\
\hline N1 & $0.56137(14)$ & $0.34515(16)$ & $0.44077(9)$ & $0.0539(4)$ \\
\hline $\mathrm{O} 1$ & $0.66753(13)$ & $0.60906(17)$ & $0.31011(10)$ & $0.0811(5)$ \\
\hline $\mathrm{O} 2$ & $0.76216(13)$ & $0.27628(19)$ & $0.30333(10)$ & $0.0914(6)$ \\
\hline $\mathrm{H} 2$ & 0.804328 & 0.226747 & 0.274357 & $0.137^{*}$ \\
\hline $\mathrm{C} 7$ & $0.43814(16)$ & $0.35514(19)$ & $0.42800(10)$ & $0.0508(4)$ \\
\hline C6 & $0.36108(18)$ & $0.2513(2)$ & $0.47105(11)$ & $0.0536(4)$ \\
\hline $\mathrm{C} 8$ & $0.38202(17)$ & $0.4630(2)$ & $0.37706(11)$ & $0.0567(5)$ \\
\hline H8 & 0.294010 & 0.471278 & 0.371699 & $0.068^{*}$ \\
\hline $\mathrm{C} 10$ & $0.58990(18)$ & $0.5384(2)$ & $0.34591(11)$ & $0.0589(5)$ \\
\hline $\mathrm{C} 12$ & 0.83446 (19) & $0.3288(2)$ & $0.36076(12)$ & $0.0612(5)$ \\
\hline $\mathrm{C} 9$ & $0.45351(17)$ & $0.5535(2)$ & $0.33636(11)$ & $0.0594(5)$ \\
\hline $\mathrm{O} 3$ & $0.94446(16)$ & 0.3027 & $0.37082(12)$ & $0.1213(7)$ \\
\hline C11 & 0.77005 (17) & $0.4220(2)$ & $0.41864(12)$ & $0.0634(5)$ \\
\hline H11A & 0.785002 & 0.382843 & 0.472680 & $0.076^{*}$ \\
\hline H11B & 0.808847 & 0.515829 & 0.418553 & $0.076^{*}$ \\
\hline C14 & 0.26299 (19) & $0.7017(2)$ & $0.29161(12)$ & $0.0638(5)$ \\
\hline $\mathrm{C} 5$ & $0.2306(2)$ & $0.2435(2)$ & $0.45719(13)$ & $0.0677(6)$ \\
\hline H5 & 0.189656 & 0.304979 & 0.419821 & $0.081 *$ \\
\hline $\mathrm{C} 15$ & $0.2264(2)$ & $0.7924(3)$ & $0.35035(15)$ & $0.0794(7)$ \\
\hline H15 & 0.288323 & 0.838285 & 0.383550 & $0.095^{*}$ \\
\hline $\mathrm{C} 4$ & $0.1600(2)$ & $0.1465(3)$ & $0.49763(14)$ & $0.0780(6)$ \\
\hline H4 & 0.072279 & 0.143929 & 0.487252 & $0.094^{*}$ \\
\hline C13 & $0.4010(2)$ & 0.6709 (3) & $0.28207(15)$ & $0.0822(7)$ \\
\hline $\mathrm{H} 13 \mathrm{~A}$ & 0.411644 & 0.644798 & 0.226260 & $0.099 *$ \\
\hline H13B & 0.449726 & 0.757252 & 0.293614 & $0.099 *$ \\
\hline $\mathrm{C} 17$ & $0.0092(2)$ & $0.7521(3)$ & $0.31546(16)$ & $0.0888(8)$ \\
\hline H17 & -0.075763 & 0.769037 & 0.323863 & $0.107^{*}$ \\
\hline C19 & $0.1676(2)$ & $0.6361(3)$ & $0.24455(14)$ & $0.0836(7)$ \\
\hline H19 & 0.188376 & 0.573613 & 0.203863 & $0.100^{*}$ \\
\hline $\mathrm{C} 3$ & $0.2156(3)$ & $0.0557(3)$ & $0.55173(15)$ & $0.0859(7)$ \\
\hline H3 & 0.167270 & -0.008738 & 0.579307 & $0.103^{*}$ \\
\hline $\mathrm{C} 1$ & $0.4167(2)$ & $0.1565(3)$ & $0.52585(16)$ & $0.0904(8)$ \\
\hline H1 & 0.504386 & 0.157532 & 0.536391 & $0.109^{*}$ \\
\hline
\end{tabular}
matrix. The cell esds are taken into account individually in the estimation of esds in distances, angles and torsion angles; correlations between esds in cell parameters are only used when they are defined by crystal symmetry. An approximate (isotropic) treatment of cell esds is used for estimating esds involving l.s. planes.

Fractional atomic coordinates and isotropic or equivalent isotropic displacement parameters $\left(\AA^{2}\right)$ 


\begin{tabular}{lllll} 
C16 & $0.1008(2)$ & $0.8173(3)$ & $0.36152(17)$ & $0.0958(8)$ \\
H16 & 0.078911 & 0.880366 & 0.401664 & $0.115^{*}$ \\
C18 & $0.0408(2)$ & $0.6619(3)$ & $0.25690(16)$ & $0.0901(8)$ \\
H18 & -0.022580 & 0.616712 & 0.224545 & $0.108^{*}$ \\
C2 & $0.3444(3)$ & $0.0599(3)$ & $0.56547(18)$ & $0.1078(10)$ \\
H2A & 0.384238 & -0.003519 & 0.602205 & $0.129^{*}$ \\
\hline
\end{tabular}

Atomic displacement parameters $\left(\AA^{2}\right)$

\begin{tabular}{lllllll}
\hline & $U^{11}$ & $U^{22}$ & $U^{33}$ & $U^{12}$ & $U^{13}$ & $U^{23}$ \\
\hline $\mathrm{N} 2$ & $0.0449(8)$ & $0.0597(10)$ & $0.0611(9)$ & $0.0062(7)$ & $0.0087(7)$ & $-0.0024(8)$ \\
$\mathrm{N} 1$ & $0.0496(9)$ & $0.0560(9)$ & $0.0565(8)$ & $0.0062(7)$ & $0.0063(7)$ & $-0.0028(7)$ \\
O1 & $0.0572(9)$ & $0.0901(11)$ & $0.0988(11)$ & $0.0021(8)$ & $0.0255(8)$ & $0.0262(9)$ \\
O2 & $0.0511(8)$ & $0.1258(14)$ & $0.0963(11)$ & $0.0206(9)$ & $-0.0021(8)$ & $-0.0447(10)$ \\
C7 & $0.0487(10)$ & $0.0515(11)$ & $0.0526(9)$ & $0.0073(8)$ & $0.0051(8)$ & $-0.0036(8)$ \\
C6 & $0.0549(11)$ & $0.0523(11)$ & $0.0542(10)$ & $0.0049(9)$ & $0.0079(8)$ & $-0.0018(8)$ \\
C8 & $0.0457(10)$ & $0.0611(11)$ & $0.0643(11)$ & $0.0097(9)$ & $0.0113(8)$ & $0.0068(9)$ \\
C10 & $0.0512(10)$ & $0.0611(12)$ & $0.0662(11)$ & $0.0069(10)$ & $0.0176(9)$ & $0.0038(10)$ \\
C12 & $0.0514(7)$ & $0.0670(9)$ & $0.0653(8)$ & $0.0051(7)$ & $0.0037(7)$ & $0.0008(7)$ \\
C9 & $0.0503(11)$ & $0.0658(12)$ & $0.0638(11)$ & $0.0123(9)$ & $0.0163(9)$ & $0.0119(9)$ \\
O3 & $0.0569(8)$ & $0.1817(15)$ & $0.1240(12)$ & $0.0325(10)$ & $-0.0028(8)$ & $-0.0424(12)$ \\
C11 & $0.0459(11)$ & $0.0735(14)$ & $0.0701(12)$ & $0.0039(9)$ & $-0.0017(9)$ & $-0.0047(10)$ \\
C14 & $0.0580(12)$ & $0.0676(13)$ & $0.0666(12)$ & $0.0121(10)$ & $0.0103(10)$ & $0.0237(10)$ \\
C5 & $0.0596(12)$ & $0.0704(14)$ & $0.0739(13)$ & $0.0014(10)$ & $0.0100(10)$ & $0.0141(10)$ \\
C15 & $0.0572(13)$ & $0.0833(16)$ & $0.0964(16)$ & $0.0031(12)$ & $-0.0039(11)$ & $-0.0097(13)$ \\
C4 & $0.0619(13)$ & $0.0798(15)$ & $0.0939(16)$ & $-0.0060(12)$ & $0.0162(11)$ & $0.0109(13)$ \\
C13 & $0.0623(13)$ & $0.0909(17)$ & $0.0962(16)$ & $0.0209(12)$ & $0.0249(11)$ & $0.0393(14)$ \\
C17 & $0.0555(13)$ & $0.119(2)$ & $0.0923(17)$ & $0.0121(14)$ & $0.0047(12)$ & $-0.0046(16)$ \\
C19 & $0.0876(18)$ & $0.0948(18)$ & $0.0684(13)$ & $0.0214(14)$ & $0.0051(12)$ & $-0.0045(13)$ \\
C3 & $0.0887(18)$ & $0.0720(15)$ & $0.0992(17)$ & $-0.0067(14)$ & $0.0234(14)$ & $0.0203(13)$ \\
C1 & $0.0660(14)$ & $0.0925(18)$ & $0.1114(19)$ & $0.0008(13)$ & $-0.0045(13)$ & $0.0438(16)$ \\
C16 & $0.0666(15)$ & $0.118(2)$ & $0.1027(18)$ & $0.0164(15)$ & $0.0038(13)$ & $-0.0330(17)$ \\
C18 & $0.0709(16)$ & $0.112(2)$ & $0.0846(16)$ & $-0.0008(15)$ & $-0.0154(12)$ & $-0.0093(15)$ \\
C2 & $0.100(2)$ & $0.100(2)$ & $0.122(2)$ & $0.0027(17)$ & $-0.0042(17)$ & $0.0588(18)$ \\
& & & & & & \\
\hline & & & & & &
\end{tabular}

Geometric parameters $\left(\AA,{ }^{\circ}\right)$

\begin{tabular}{llll}
\hline $\mathrm{N} 2-\mathrm{N} 1$ & $1.353(2)$ & $\mathrm{C} 14-\mathrm{C} 13$ & $1.503(3)$ \\
$\mathrm{N} 2-\mathrm{C} 10$ & $1.371(2)$ & $\mathrm{C} 5-\mathrm{C} 4$ & $1.377(3)$ \\
$\mathrm{N} 2-\mathrm{C} 11$ & $1.458(2)$ & $\mathrm{C} 5-\mathrm{H} 5$ & 0.9300 \\
$\mathrm{~N} 1-\mathrm{C} 7$ & $1.306(2)$ & $\mathrm{C} 15-\mathrm{C} 16$ & $1.371(3)$ \\
$\mathrm{O} 1-\mathrm{C} 10$ & $1.235(2)$ & $\mathrm{C} 15-\mathrm{H} 15$ & 0.9300 \\
$\mathrm{O} 2-\mathrm{C} 12$ & $1.277(2)$ & $\mathrm{C} 4-\mathrm{C} 3$ & $1.341(3)$ \\
$\mathrm{O} 2-\mathrm{H} 2$ & 0.8200 & $\mathrm{C} 4-\mathrm{H} 4$ & 0.9300 \\
$\mathrm{C} 7-\mathrm{C} 8$ & $1.420(2)$ & $\mathrm{C} 13-\mathrm{H} 13 \mathrm{~A}$ & 0.9700 \\
$\mathrm{C} 7-\mathrm{C} 6$ & $1.481(3)$ & $\mathrm{C} 13-\mathrm{H} 13 \mathrm{~B}$ & 0.9700 \\
$\mathrm{C} 6-\mathrm{C} 1$ & $1.373(3)$ & $\mathrm{C} 17-\mathrm{C} 16$ & $1.337(3)$ \\
$\mathrm{C} 6-\mathrm{C} 5$ & $1.382(3)$ & $\mathrm{C} 17-\mathrm{C} 18$ & $1.345(4)$
\end{tabular}




\begin{tabular}{|c|c|c|c|}
\hline $\mathrm{C} 8-\mathrm{C} 9$ & $1.346(3)$ & $\mathrm{C} 17-\mathrm{H} 17$ & 0.9300 \\
\hline $\mathrm{C} 8-\mathrm{H} 8$ & 0.9300 & $\mathrm{C} 19-\mathrm{C} 18$ & $1.389(3)$ \\
\hline $\mathrm{C} 10-\mathrm{C} 9$ & $1.444(3)$ & C19-H19 & 0.9300 \\
\hline $\mathrm{C} 12-\mathrm{O} 3$ & $1.187(2)$ & $\mathrm{C} 3-\mathrm{C} 2$ & $1.363(4)$ \\
\hline $\mathrm{C} 12-\mathrm{C} 11$ & $1.494(3)$ & $\mathrm{C} 3-\mathrm{H} 3$ & 0.9300 \\
\hline $\mathrm{C} 9-\mathrm{C} 13$ & $1.502(3)$ & $\mathrm{C} 1-\mathrm{C} 2$ & $1.378(4)$ \\
\hline $\mathrm{C} 11-\mathrm{H} 11 \mathrm{~A}$ & 0.9700 & $\mathrm{C} 1-\mathrm{H} 1$ & 0.9300 \\
\hline C11-H11B & 0.9700 & $\mathrm{C} 16-\mathrm{H} 16$ & 0.9300 \\
\hline $\mathrm{C} 14-\mathrm{C} 15$ & $1.367(3)$ & C18-H18 & 0.9300 \\
\hline $\mathrm{C} 14-\mathrm{C} 19$ & $1.375(3)$ & $\mathrm{C} 2-\mathrm{H} 2 \mathrm{~A}$ & 0.9300 \\
\hline $\mathrm{N} 1-\mathrm{N} 2-\mathrm{C} 10$ & $126.22(15)$ & $\mathrm{C} 6-\mathrm{C} 5-\mathrm{H} 5$ & 119.3 \\
\hline $\mathrm{N} 1-\mathrm{N} 2-\mathrm{C} 11$ & $114.74(15)$ & $\mathrm{C} 14-\mathrm{C} 15-\mathrm{C} 16$ & $121.6(2)$ \\
\hline $\mathrm{C} 10-\mathrm{N} 2-\mathrm{C} 11$ & $119.00(16)$ & $\mathrm{C} 14-\mathrm{C} 15-\mathrm{H} 15$ & 119.2 \\
\hline $\mathrm{C} 7-\mathrm{N} 1-\mathrm{N} 2$ & $117.48(15)$ & $\mathrm{C} 16-\mathrm{C} 15-\mathrm{H} 15$ & 119.2 \\
\hline $\mathrm{C} 12-\mathrm{O} 2-\mathrm{H} 2$ & 109.5 & $\mathrm{C} 3-\mathrm{C} 4-\mathrm{C} 5$ & $121.1(2)$ \\
\hline $\mathrm{N} 1-\mathrm{C} 7-\mathrm{C} 8$ & $121.17(17)$ & $\mathrm{C} 3-\mathrm{C} 4-\mathrm{H} 4$ & 119.4 \\
\hline $\mathrm{N} 1-\mathrm{C} 7-\mathrm{C} 6$ & $116.60(16)$ & $\mathrm{C} 5-\mathrm{C} 4-\mathrm{H} 4$ & 119.4 \\
\hline $\mathrm{C} 8-\mathrm{C} 7-\mathrm{C} 6$ & $122.21(16)$ & $\mathrm{C} 9-\mathrm{C} 13-\mathrm{C} 14$ & $113.43(17)$ \\
\hline $\mathrm{C} 1-\mathrm{C} 6-\mathrm{C} 5$ & $116.86(19)$ & $\mathrm{C} 9-\mathrm{C} 13-\mathrm{H} 13 \mathrm{~A}$ & 108.9 \\
\hline $\mathrm{C} 1-\mathrm{C} 6-\mathrm{C} 7$ & $121.27(18)$ & $\mathrm{C} 14-\mathrm{C} 13-\mathrm{H} 13 \mathrm{~A}$ & 108.9 \\
\hline $\mathrm{C} 5-\mathrm{C} 6-\mathrm{C} 7$ & $121.86(17)$ & $\mathrm{C} 9-\mathrm{C} 13-\mathrm{H} 13 \mathrm{~B}$ & 108.9 \\
\hline $\mathrm{C} 9-\mathrm{C} 8-\mathrm{C} 7$ & $121.38(17)$ & $\mathrm{C} 14-\mathrm{C} 13-\mathrm{H} 13 \mathrm{~B}$ & 108.9 \\
\hline $\mathrm{C} 9-\mathrm{C} 8-\mathrm{H} 8$ & 119.3 & $\mathrm{H} 13 \mathrm{~A}-\mathrm{C} 13-\mathrm{H} 13 \mathrm{~B}$ & 107.7 \\
\hline $\mathrm{C} 7-\mathrm{C} 8-\mathrm{H} 8$ & 119.3 & $\mathrm{C} 16-\mathrm{C} 17-\mathrm{C} 18$ & $119.6(2)$ \\
\hline $\mathrm{O} 1-\mathrm{C} 10-\mathrm{N} 2$ & $119.04(17)$ & $\mathrm{C} 16-\mathrm{C} 17-\mathrm{H} 17$ & 120.2 \\
\hline $\mathrm{O} 1-\mathrm{C} 10-\mathrm{C} 9$ & 125.67 (19) & $\mathrm{C} 18-\mathrm{C} 17-\mathrm{H} 17$ & 120.2 \\
\hline $\mathrm{N} 2-\mathrm{C} 10-\mathrm{C} 9$ & $115.28(16)$ & $\mathrm{C} 14-\mathrm{C} 19-\mathrm{C} 18$ & $120.9(2)$ \\
\hline $\mathrm{O} 3-\mathrm{C} 12-\mathrm{O} 2$ & $123.7(2)$ & C14-C19-H19 & 119.5 \\
\hline $\mathrm{O} 3-\mathrm{C} 12-\mathrm{C} 11$ & $120.9(2)$ & $\mathrm{C} 18-\mathrm{C} 19-\mathrm{H} 19$ & 119.5 \\
\hline $\mathrm{O} 2-\mathrm{C} 12-\mathrm{C} 11$ & $115.36(17)$ & $\mathrm{C} 4-\mathrm{C} 3-\mathrm{C} 2$ & $118.6(2)$ \\
\hline $\mathrm{C} 8-\mathrm{C} 9-\mathrm{C} 10$ & $118.14(18)$ & $\mathrm{C} 4-\mathrm{C} 3-\mathrm{H} 3$ & 120.7 \\
\hline $\mathrm{C} 8-\mathrm{C} 9-\mathrm{C} 13$ & $124.38(17)$ & $\mathrm{C} 2-\mathrm{C} 3-\mathrm{H} 3$ & 120.7 \\
\hline $\mathrm{C} 10-\mathrm{C} 9-\mathrm{C} 13$ & $117.48(17)$ & $\mathrm{C} 6-\mathrm{C} 1-\mathrm{C} 2$ & $121.0(2)$ \\
\hline $\mathrm{N} 2-\mathrm{C} 11-\mathrm{C} 12$ & $114.75(16)$ & $\mathrm{C} 6-\mathrm{C} 1-\mathrm{H} 1$ & 119.5 \\
\hline $\mathrm{N} 2-\mathrm{C} 11-\mathrm{H} 11 \mathrm{~A}$ & 108.6 & $\mathrm{C} 2-\mathrm{C} 1-\mathrm{H} 1$ & 119.5 \\
\hline $\mathrm{C} 12-\mathrm{C} 11-\mathrm{H} 11 \mathrm{~A}$ & 108.6 & $\mathrm{C} 17-\mathrm{C} 16-\mathrm{C} 15$ & $120.9(2)$ \\
\hline $\mathrm{N} 2-\mathrm{C} 11-\mathrm{H} 11 \mathrm{~B}$ & 108.6 & $\mathrm{C} 17-\mathrm{C} 16-\mathrm{H} 16$ & 119.6 \\
\hline $\mathrm{C} 12-\mathrm{C} 11-\mathrm{H} 11 \mathrm{~B}$ & 108.6 & $\mathrm{C} 15-\mathrm{C} 16-\mathrm{H} 16$ & 119.6 \\
\hline $\mathrm{H} 11 \mathrm{~A}-\mathrm{C} 11-\mathrm{H} 11 \mathrm{~B}$ & 107.6 & $\mathrm{C} 17-\mathrm{C} 18-\mathrm{C} 19$ & $120.3(2)$ \\
\hline $\mathrm{C} 15-\mathrm{C} 14-\mathrm{C} 19$ & $116.8(2)$ & $\mathrm{C} 17-\mathrm{C} 18-\mathrm{H} 18$ & 119.9 \\
\hline $\mathrm{C} 15-\mathrm{C} 14-\mathrm{C} 13$ & $121.1(2)$ & $\mathrm{C} 19-\mathrm{C} 18-\mathrm{H} 18$ & 119.9 \\
\hline $\mathrm{C} 19-\mathrm{C} 14-\mathrm{C} 13$ & $122.1(2)$ & $\mathrm{C} 3-\mathrm{C} 2-\mathrm{C} 1$ & $121.1(2)$ \\
\hline $\mathrm{C} 4-\mathrm{C} 5-\mathrm{C} 6$ & $121.3(2)$ & $\mathrm{C} 3-\mathrm{C} 2-\mathrm{H} 2 \mathrm{~A}$ & 119.4 \\
\hline $\mathrm{C} 4-\mathrm{C} 5-\mathrm{H} 5$ & 119.3 & $\mathrm{C} 1-\mathrm{C} 2-\mathrm{H} 2 \mathrm{~A}$ & 119.4 \\
\hline
\end{tabular}


Hydrogen-bond geometry $\left(\AA,{ }^{\circ}\right)$

\begin{tabular}{lllll}
\hline$D-\mathrm{H} \cdots A$ & $D-\mathrm{H}$ & $\mathrm{H} \cdots A$ & $D \cdots A$ & $D-\mathrm{H} \cdots A$ \\
\hline $\mathrm{O} 2-\mathrm{H} 2 \cdots \mathrm{O} 1^{\mathrm{i}}$ & 0.82 & 1.82 & $2.593(2)$ & 156 \\
$\mathrm{C} 1-\mathrm{H} 1 \cdots \mathrm{N} 1$ & 0.93 & 2.47 & $2.780(3)$ & 100 \\
\hline
\end{tabular}

Symmetry code: (i) $-x+3 / 2, y-1 / 2,-z+1 / 2$.

(II)

\section{Crystal data}

$\mathrm{C}_{19} \mathrm{H}_{16} \mathrm{~N}_{2} \mathrm{O}_{3}$

$M_{r}=320.34$

Monoclinic, $P 2_{1} / c$

$a=10.5976(6) \AA$

$b=15.5500(7) \AA$

$c=10.3731(7) \AA$

$\beta=109.120(5)^{\circ}$

$V=1615.11(17) \AA^{3}$

$Z=4$

\section{Data collection}

STOE IPDS 2

diffractometer

Radiation source: sealed X-ray tube, $12 \times 0.4$ $\mathrm{mm}$ long-fine focus

Plane graphite monochromator

Detector resolution: 6.67 pixels $\mathrm{mm}^{-1}$

rotation method scans

Absorption correction: integration

(X-RED32; Stoe \& Cie, 2002)

\section{Refinement}

Refinement on $F^{2}$

Least-squares matrix: full

$R\left[F^{2}>2 \sigma\left(F^{2}\right)\right]=0.049$

$w R\left(F^{2}\right)=0.131$

$S=0.98$

4562 reflections

218 parameters

0 restraints
$F(000)=672$

$D_{\mathrm{x}}=1.317 \mathrm{Mg} \mathrm{m}^{-3}$

Mo $K \alpha$ radiation, $\lambda=0.71073 \AA$

Cell parameters from 11065 reflections

$\theta=2.0-30.2^{\circ}$

$\mu=0.09 \mathrm{~mm}^{-1}$

$T=296 \mathrm{~K}$

Prism, colorless

$0.77 \times 0.70 \times 0.59 \mathrm{~mm}$

$T_{\min }=0.950, T_{\max }=0.966$

12114 measured reflections

4562 independent reflections

2560 reflections with $I>2 \sigma(I)$

$R_{\text {int }}=0.037$

$\theta_{\max }=29.8^{\circ}, \theta_{\min }=2.0^{\circ}$

$h=-14 \rightarrow 14$

$k=-21 \rightarrow 21$

$l=-9 \rightarrow 14$

Hydrogen site location: inferred from

neighbouring sites

$\mathrm{H}$-atom parameters constrained

$w=1 /\left[\sigma^{2}\left(F_{\mathrm{o}}^{2}\right)+(0.0658 P)^{2}\right]$

where $P=\left(F_{\mathrm{o}}{ }^{2}+2 F_{\mathrm{c}}{ }^{2}\right) / 3$

$(\Delta / \sigma)_{\max }<0.001$

$\Delta \rho_{\max }=0.21$ e $\AA^{-3}$

$\Delta \rho_{\min }=-0.21 \mathrm{e} \AA^{-3}$

Special details

Geometry. All esds (except the esd in the dihedral angle between two 1.s. planes) are estimated using the full covariance matrix. The cell esds are taken into account individually in the estimation of esds in distances, angles and torsion angles; correlations between esds in cell parameters are only used when they are defined by crystal symmetry. An approximate (isotropic) treatment of cell esds is used for estimating esds involving l.s. planes.

Fractional atomic coordinates and isotropic or equivalent isotropic displacement parameters $\left(\AA^{2}\right)$

\begin{tabular}{lllll}
\hline & $x$ & $y$ & $z$ & $U_{\text {iso }} * U_{\text {eq }}$ \\
\hline O1 & $0.72419(10)$ & $0.27629(8)$ & $0.59311(12)$ & $0.0685(3)$ \\
O3 & $0.85719(10)$ & $0.46576(8)$ & $0.50173(13)$ & $0.0697(3)$ \\
O2 & $0.97177(11)$ & $0.41088(9)$ & $0.37548(13)$ & $0.0746(4)$
\end{tabular}




\begin{tabular}{|c|c|c|c|c|}
\hline H3 & 1.024031 & 0.449746 & 0.410547 & $0.112 *$ \\
\hline N1 & $0.56525(10)$ & $0.40450(8)$ & $0.30448(12)$ & 0.0469 (3) \\
\hline N2 & $0.65255(10)$ & $0.35304(8)$ & $0.39742(12)$ & $0.0482(3)$ \\
\hline $\mathrm{C} 7$ & $0.45128(12)$ & $0.42124(9)$ & $0.32229(14)$ & $0.0448(3)$ \\
\hline $\mathrm{C} 10$ & $0.63572(13)$ & $0.31919(10)$ & $0.51338(15)$ & $0.0505(3)$ \\
\hline $\mathrm{C} 8$ & $0.41953(13)$ & $0.38747(10)$ & $0.43611(15)$ & $0.0518(3)$ \\
\hline H8 & 0.336938 & 0.399584 & 0.445235 & $0.062 *$ \\
\hline C6 & $0.35817(13)$ & $0.47589(9)$ & $0.21629(14)$ & $0.0467(3)$ \\
\hline C9 & $0.50740(13)$ & $0.33851(10)$ & $0.52997(15)$ & $0.0510(3)$ \\
\hline $\mathrm{C} 12$ & $0.87184(13)$ & $0.41235(11)$ & $0.42288(15)$ & $0.0540(4)$ \\
\hline C11 & $0.77702(13)$ & $0.33946(10)$ & $0.37033(16)$ & $0.0529(4)$ \\
\hline H11A & 0.758711 & 0.333700 & 0.272786 & $0.064 *$ \\
\hline H11B & 0.817891 & 0.286421 & 0.413433 & $0.064^{*}$ \\
\hline C14 & $0.34689(14)$ & $0.30038(11)$ & $0.65991(16)$ & $0.0559(4)$ \\
\hline $\mathrm{C} 1$ & $0.37838(15)$ & $0.48952(12)$ & $0.09292(17)$ & $0.0629(4)$ \\
\hline H1 & 0.450530 & 0.463405 & 0.076387 & $0.076^{*}$ \\
\hline $\mathrm{C} 13$ & $0.48712(15)$ & $0.29980(13)$ & $0.65443(18)$ & $0.0679(5)$ \\
\hline $\mathrm{H} 13 \mathrm{~A}$ & 0.543928 & 0.330261 & 0.733863 & $0.082 *$ \\
\hline H13B & 0.517681 & 0.240631 & 0.661889 & $0.082 *$ \\
\hline $\mathrm{C} 2$ & $0.29390(17)$ & $0.54093(13)$ & $-0.00593(19)$ & $0.0744(5)$ \\
\hline $\mathrm{H} 2$ & 0.309754 & 0.549564 & -0.088011 & $0.089 *$ \\
\hline $\mathrm{C} 5$ & $0.25091(17)$ & $0.51563(13)$ & $0.23724(19)$ & $0.0733(5)$ \\
\hline H5 & 0.234983 & 0.508074 & 0.319510 & $0.088^{*}$ \\
\hline $\mathrm{C} 3$ & $0.18788(19)$ & $0.57899(13)$ & 0.0157 (2) & $0.0814(6)$ \\
\hline $\mathrm{H} 3 \mathrm{~A}$ & 0.129995 & 0.613227 & -0.051425 & $0.098 *$ \\
\hline C19 & $0.3040(2)$ & $0.36098(15)$ & $0.7325(2)$ & $0.0846(6)$ \\
\hline H19 & 0.361300 & 0.405216 & 0.775869 & $0.102 *$ \\
\hline $\mathrm{C} 15$ & $0.25885(18)$ & $0.23814(14)$ & $0.5959(2)$ & $0.0837(6)$ \\
\hline H15 & 0.283752 & 0.197158 & 0.543528 & $0.100^{*}$ \\
\hline $\mathrm{C} 17$ & 0.09285 (19) & $0.29337(18)$ & $0.6803(3)$ & $0.0941(7)$ \\
\hline H17 & 0.008047 & 0.290075 & 0.688145 & $0.113^{*}$ \\
\hline $\mathrm{C} 4$ & $0.1669(2)$ & $0.56655(15)$ & $0.1373(2)$ & $0.0960(7)$ \\
\hline $\mathrm{H} 4$ & 0.094447 & 0.592977 & 0.152843 & $0.115^{*}$ \\
\hline $\mathrm{C} 18$ & $0.1777(2)$ & $0.35732(18)$ & $0.7422(3)$ & $0.1008(7)$ \\
\hline H18 & 0.150476 & 0.399081 & 0.791625 & $0.121^{*}$ \\
\hline $\mathrm{C} 16$ & $0.1334(2)$ & $0.23488(17)$ & $0.6074(3)$ & $0.1056(8)$ \\
\hline H16 & 0.075399 & 0.191088 & 0.563708 & $0.127 *$ \\
\hline
\end{tabular}

Atomic displacement parameters $\left(\AA^{2}\right)$

\begin{tabular}{lllllll}
\hline & $U^{11}$ & $U^{22}$ & $U^{33}$ & $U^{12}$ & $U^{13}$ & $U^{23}$ \\
\hline O1 & $0.0598(6)$ & $0.0850(8)$ & $0.0606(7)$ & $0.0170(6)$ & $0.0196(5)$ & $0.0211(6)$ \\
O3 & $0.0610(6)$ & $0.0868(8)$ & $0.0744(8)$ & $-0.0176(6)$ & $0.0402(6)$ & $-0.0276(6)$ \\
O2 & $0.0615(6)$ & $0.1001(10)$ & $0.0777(8)$ & $-0.0164(6)$ & $0.0437(6)$ & $-0.0276(7)$ \\
N1 & $0.0482(5)$ & $0.0524(7)$ & $0.0415(6)$ & $0.0005(5)$ & $0.0167(5)$ & $0.0007(5)$ \\
N2 & $0.0435(5)$ & $0.0561(7)$ & $0.0464(7)$ & $0.0002(5)$ & $0.0166(5)$ & $-0.0001(5)$ \\
C7 & $0.0457(6)$ & $0.0494(8)$ & $0.0417(7)$ & $-0.0021(6)$ & $0.0175(5)$ & $-0.0003(6)$ \\
C10 & $0.0493(7)$ & $0.0556(9)$ & $0.0462(8)$ & $-0.0019(6)$ & $0.0152(6)$ & $0.0019(7)$
\end{tabular}




\begin{tabular}{lllllll} 
C8 & $0.0466(6)$ & $0.0643(9)$ & $0.0485(8)$ & $0.0003(6)$ & $0.0212(6)$ & $0.0065(7)$ \\
C6 & $0.0499(7)$ & $0.0503(8)$ & $0.0442(7)$ & $0.0014(6)$ & $0.0212(6)$ & $0.0016(6)$ \\
C9 & $0.0495(7)$ & $0.0586(9)$ & $0.0467(8)$ & $-0.0039(6)$ & $0.0180(6)$ & $0.0058(7)$ \\
C12 & $0.0459(7)$ & $0.0737(10)$ & $0.0471(8)$ & $0.0002(7)$ & $0.0218(6)$ & $-0.0021(7)$ \\
C11 & $0.0467(7)$ & $0.0654(10)$ & $0.0497(8)$ & $0.0037(6)$ & $0.0200(6)$ & $-0.0034(7)$ \\
C14 & $0.0561(7)$ & $0.0661(10)$ & $0.0481(8)$ & $0.0005(7)$ & $0.0207(6)$ & $0.0174(7)$ \\
C1 & $0.0595(8)$ & $0.0819(12)$ & $0.0563(9)$ & $0.0189(8)$ & $0.0310(7)$ & $0.0177(8)$ \\
C13 & $0.0581(8)$ & $0.0897(13)$ & $0.0592(10)$ & $0.0047(8)$ & $0.0236(7)$ & $0.0264(9)$ \\
C2 & $0.0784(10)$ & $0.0946(13)$ & $0.0575(10)$ & $0.0234(10)$ & $0.0323(8)$ & $0.0246(9)$ \\
C5 & $0.0790(10)$ & $0.0930(13)$ & $0.0618(10)$ & $0.0284(9)$ & $0.0419(9)$ & $0.0175(9)$ \\
C3 & $0.0832(11)$ & $0.0951(15)$ & $0.0701(12)$ & $0.0376(10)$ & $0.0307(9)$ & $0.0296(10)$ \\
C19 & $0.0799(11)$ & $0.0973(15)$ & $0.0831(14)$ & $-0.0166(10)$ & $0.0355(10)$ & $-0.0194(11)$ \\
C15 & $0.0728(11)$ & $0.0753(13)$ & $0.1021(16)$ & $-0.0058(10)$ & $0.0273(10)$ & $-0.0127(11)$ \\
C17 & $0.0596(10)$ & $0.138(2)$ & $0.0901(16)$ & $-0.0055(13)$ & $0.0324(10)$ & $0.0234(15)$ \\
C4 & $0.0908(12)$ & $0.1224(18)$ & $0.0917(15)$ & $0.0572(13)$ & $0.0528(11)$ & $0.0360(13)$ \\
C18 & $0.0848(13)$ & $0.140(2)$ & $0.0911(16)$ & $0.0060(14)$ & $0.0478(12)$ & $-0.0147(15)$ \\
C16 & $0.0719(12)$ & $0.0996(17)$ & $0.138(2)$ & $-0.0280(12)$ & $0.0244(13)$ & $-0.0066(16)$ \\
& & & & & & \\
\hline
\end{tabular}

Geometric parameters $\left(\AA,{ }^{\circ}\right)$

\begin{tabular}{llll}
\hline $\mathrm{O} 1-\mathrm{C} 10$ & $1.2249(17)$ & $\mathrm{C} 14-\mathrm{C} 13$ & $1.506(2)$ \\
$\mathrm{O} 3-\mathrm{C} 12$ & $1.2108(18)$ & $\mathrm{C} 1-\mathrm{C} 2$ & $1.375(2)$ \\
$\mathrm{O} 2-\mathrm{C} 12$ & $1.3057(16)$ & $\mathrm{C} 1-\mathrm{H} 1$ & 0.9300 \\
$\mathrm{O} 2-\mathrm{H} 3$ & 0.8200 & $\mathrm{C} 13-\mathrm{H} 13 \mathrm{~A}$ & 0.9700 \\
$\mathrm{~N} 1-\mathrm{C} 7$ & $1.3064(16)$ & $\mathrm{C} 13-\mathrm{H} 13 \mathrm{~B}$ & 0.9700 \\
$\mathrm{~N} 1-\mathrm{N} 2$ & $1.3570(16)$ & $\mathrm{C} 2-\mathrm{C} 3$ & $1.352(2)$ \\
$\mathrm{N} 2-\mathrm{C} 10$ & $1.3771(18)$ & $\mathrm{C} 2-\mathrm{H} 2$ & 0.9300 \\
$\mathrm{~N} 2-\mathrm{C} 11$ & $1.4513(17)$ & $\mathrm{C} 5-\mathrm{C} 4$ & $1.375(3)$ \\
$\mathrm{C} 7-\mathrm{C} 8$ & $1.4294(19)$ & $\mathrm{C} 5-\mathrm{H} 5$ & 0.9300 \\
$\mathrm{C} 7-\mathrm{C} 6$ & $1.4813(19)$ & $\mathrm{C} 3-\mathrm{C} 4$ & $1.364(3)$ \\
$\mathrm{C} 10-\mathrm{C} 9$ & $1.4568(19)$ & $\mathrm{C} 3-\mathrm{H} 3 \mathrm{~A}$ & 0.9300 \\
$\mathrm{C} 8-\mathrm{C} 9$ & $1.342(2)$ & $\mathrm{C} 19-\mathrm{C} 18$ & $1.376(3)$ \\
$\mathrm{C} 8-\mathrm{H} 8$ & 0.9300 & $\mathrm{C} 19-\mathrm{H} 19$ & 0.9300 \\
$\mathrm{C} 6-\mathrm{C} 5$ & $1.372(2)$ & $\mathrm{C} 15-\mathrm{C} 16$ & $1.374(3)$ \\
$\mathrm{C} 6-\mathrm{C} 1$ & $1.381(2)$ & $\mathrm{C} 15-\mathrm{H} 15$ & 0.9300 \\
$\mathrm{C} 9-\mathrm{C} 13$ & $1.502(2)$ & $\mathrm{C} 17-\mathrm{C} 16$ & $1.340(4)$ \\
$\mathrm{C} 12-\mathrm{C} 11$ & $1.494(2)$ & $\mathrm{C} 17-\mathrm{C} 18$ & $1.354(3)$ \\
$\mathrm{C} 11-\mathrm{H} 11 \mathrm{~A}$ & 0.9700 & $\mathrm{C} 17-\mathrm{H} 17$ & 0.9300 \\
$\mathrm{C} 11-\mathrm{H} 11 \mathrm{~B}$ & 0.9700 & $\mathrm{C} 4-\mathrm{H} 4$ & 0.9300 \\
$\mathrm{C} 14-\mathrm{C} 15$ & $1.357(2)$ & $\mathrm{C} 18-\mathrm{H} 18$ & 0.9300 \\
$\mathrm{C} 14-\mathrm{C} 19$ & $1.373(3)$ & $\mathrm{C} 16-\mathrm{H} 16$ & 0.9300 \\
& & & 119.4 \\
$\mathrm{C} 12-\mathrm{O} 2-\mathrm{H} 3$ & 109.5 & $\mathrm{C} 6-\mathrm{C} 1-\mathrm{H} 1$ & $116.80(13)$ \\
$\mathrm{C} 7-\mathrm{N} 1-\mathrm{N} 2$ & $117.63(11)$ & $\mathrm{C} 9-\mathrm{C} 13-\mathrm{C} 14$ & 108.1 \\
$\mathrm{~N} 1-\mathrm{N} 2-\mathrm{C} 10$ & $126.35(11)$ & $\mathrm{C} 9-\mathrm{C} 13-\mathrm{H} 13 \mathrm{~A}$ & 108.1 \\
$\mathrm{~N} 1-\mathrm{N} 2-\mathrm{C} 11$ & $113.56(11)$ & $\mathrm{C} 14-\mathrm{C} 13-\mathrm{H} 13 \mathrm{~A}$ & 108.1 \\
$\mathrm{C} 10-\mathrm{N} 2-\mathrm{C} 11$ & $119.97(12)$ & $\mathrm{C} 9-\mathrm{C} 13-\mathrm{H} 13 \mathrm{~B}$ & \\
$\mathrm{~N} 1-\mathrm{C} 7-\mathrm{C} 8$ & $121.48(12)$ & $\mathrm{C} 14-\mathrm{C} 13-\mathrm{H} 13 \mathrm{~B}$ &
\end{tabular}




$\begin{array}{llll}\mathrm{N} 1-\mathrm{C} 7-\mathrm{C} 6 & 115.55(12) & \mathrm{H} 13 \mathrm{~A}-\mathrm{C} 13-\mathrm{H} 13 \mathrm{~B} & 107.3 \\ \mathrm{C} 8-\mathrm{C} 7-\mathrm{C} 6 & 122.96(11) & \mathrm{C} 3-\mathrm{C} 2-\mathrm{C} 1 & 120.27(16) \\ \mathrm{O} 1-\mathrm{C} 10-\mathrm{N} 2 & 120.56(12) & \mathrm{C} 3-\mathrm{C} 2-\mathrm{H} 2 & 119.9 \\ \mathrm{O} 1-\mathrm{C} 10-\mathrm{C} 9 & 124.76(13) & \mathrm{C} 1-\mathrm{C} 2-\mathrm{H} 2 & 119.9 \\ \mathrm{~N} 2-\mathrm{C} 10-\mathrm{C} 9 & 114.67(12) & \mathrm{C} 6-\mathrm{C} 5-\mathrm{C} 4 & 120.37(16) \\ \mathrm{C} 9-\mathrm{C} 8-\mathrm{C} 7 & 120.84(12) & \mathrm{C} 6-\mathrm{C} 5-\mathrm{H} 5 & 119.8 \\ \mathrm{C} 9-\mathrm{C} 8-\mathrm{H} 8 & 119.6 & \mathrm{C} 4-\mathrm{C} 5-\mathrm{H} 5 & 119.8 \\ \mathrm{C} 7-\mathrm{C} 8-\mathrm{H} 8 & 119.6 & \mathrm{C} 2-\mathrm{C} 3-\mathrm{C} 4 & 119.18(17) \\ \mathrm{C} 5-\mathrm{C} 6-\mathrm{C} 1 & 117.74(14) & \mathrm{C} 2-\mathrm{C} 3-\mathrm{H} 3 \mathrm{~A} & 120.4 \\ \mathrm{C} 5-\mathrm{C} 6-\mathrm{C} 7 & 121.72(13) & \mathrm{C} 4-\mathrm{C} 3-\mathrm{H} 3 \mathrm{~A} & 120.4 \\ \mathrm{C} 1-\mathrm{C} 6-\mathrm{C} 7 & 120.53(12) & \mathrm{C} 14-\mathrm{C} 19-\mathrm{C} 18 & 121.1(2) \\ \mathrm{C} 8-\mathrm{C} 9-\mathrm{C} 10 & 118.94(13) & \mathrm{C} 14-\mathrm{C} 19-\mathrm{H} 19 & 119.5 \\ \mathrm{C} 8-\mathrm{C} 9-\mathrm{C} 13 & 126.17(13) & \mathrm{C} 18-\mathrm{C} 19-\mathrm{H} 19 & 119.5 \\ \mathrm{C} 10-\mathrm{C} 9-\mathrm{C} 13 & 114.88(12) & \mathrm{C} 14-\mathrm{C} 15-\mathrm{C} 16 & 119.9 \\ \mathrm{O} 2-\mathrm{C} 12-\mathrm{O} 3 & 124.45(14) & \mathrm{C} 14-\mathrm{C} 15-\mathrm{H} 15 & 119.6 \\ \mathrm{O} 3-\mathrm{C} 12-\mathrm{C} 11 & 123.16(12) & \mathrm{C} 16-\mathrm{C} 15-\mathrm{H} 15 & 118.69(19) \\ \mathrm{O} 2-\mathrm{C} 12-\mathrm{C} 11 & 112.38(13) & \mathrm{C} 16-\mathrm{C} 17-\mathrm{C} 18 & 120.7 \\ \mathrm{~N} 2-\mathrm{C} 11-\mathrm{C} 12 & 111.36(12) & \mathrm{C} 16-\mathrm{C} 17-\mathrm{H} 17 & 120.7 \\ \mathrm{~N} 2-\mathrm{C} 11-\mathrm{H} 11 \mathrm{~A} & 109.4 & \mathrm{C} 18-\mathrm{C} 17-\mathrm{H} 17 & 121.14(16) \\ \mathrm{C} 12-\mathrm{C} 11-\mathrm{H} 11 \mathrm{~A} & 109.4 & \mathrm{C} 3-\mathrm{C} 4-\mathrm{C} 5 & 119.4 \\ \mathrm{~N} 2-\mathrm{C} 11-\mathrm{H} 11 \mathrm{~B} & 109.4 & \mathrm{C} 3-\mathrm{C} 4-\mathrm{H} 4 & 119.4 \\ \mathrm{C} 12-\mathrm{C} 11-\mathrm{H} 11 \mathrm{~B} & 109.4 & \mathrm{C} 5-\mathrm{C} 4-\mathrm{H} 4 & 120.5(2) \\ \mathrm{H} 11 \mathrm{~A}-\mathrm{C} 11-\mathrm{H} 11 \mathrm{~B} & 108.0 & \mathrm{C} 17-\mathrm{C} 18-\mathrm{C} 19 & 119.8 \\ \mathrm{C} 15-\mathrm{C} 14-\mathrm{C} 19 & 117.36(16) & \mathrm{C} 17-\mathrm{C} 18-\mathrm{H} 18 & 119.8 \\ \mathrm{C} 15-\mathrm{C} 14-\mathrm{C} 13 & 120.40(17) & \mathrm{C} 19-\mathrm{C} 18-\mathrm{H} 18 & 119.3 \\ \mathrm{C} 19-\mathrm{C} 14-\mathrm{C} 13 & 122.19(17) & \mathrm{C} 17-\mathrm{C} 16-\mathrm{C} 15 & \\ \mathrm{C} 2-\mathrm{C} 1-\mathrm{C} 6 & 121.30(14) & \mathrm{C} 17-\mathrm{C} 16-\mathrm{H} 16 & \\ \mathrm{C} 2-\mathrm{C} 1-\mathrm{H} 1 & 119.4 & \mathrm{C} 15-\mathrm{C} 16-\mathrm{H} 16 & \\ & & & \end{array}$

Hydrogen-bond geometry $\left(\AA,{ }^{\circ}\right)$

\begin{tabular}{lllll}
\hline$D-\mathrm{H} \cdots A$ & $D-\mathrm{H}$ & $\mathrm{H} \cdots A$ & $D \cdots A$ & $D-\mathrm{H} \cdots A$ \\
\hline $\mathrm{C} 11-\mathrm{H} 11 B^{\cdots} \cdots \mathrm{O} 1$ & 0.97 & 2.39 & $2.7325(19)$ & 100 \\
$\mathrm{O} 2-\mathrm{H} 3 \cdots 3^{\mathrm{i}}$ & 0.82 & 1.84 & $2.6599(16)$ & 177 \\
$\mathrm{C} 5-\mathrm{H} 5 \cdots 3^{\mathrm{ii}}$ & 0.93 & 2.40 & $3.280(2)$ & 159 \\
$\mathrm{C} 11-\mathrm{H} 11{ }^{\cdots} \cdots{ }^{\mathrm{iii}}$ & 0.97 & 2.47 & $3.2814(19)$ & 141 \\
\hline
\end{tabular}

Symmetry codes: (i) $-x+2,-y+1,-z+1$; (ii) $-x+1,-y+1,-z+1$; (iii) $x,-y+1 / 2, z-1 / 2$. 